\title{
Quand les adolescents passent à l'action en matière de santé environnementale
}

Nathalie Robitaille

\author{
(2) OpenEdition \\ Journals \\ Édition électronique \\ URL : http://journals.openedition.org/ere/533 \\ DOI : $10.4000 /$ ere.533 \\ ISSN : 2561-2271 \\ Éditeur \\ Centr'ERE
}

Référence électronique

Nathalie Robitaille, «Quand les adolescents passent à l'action en matière de santé environnementale ", Éducation relative à l'environnement [En ligne], Volume 12 | 2015, mis en ligne le 20 mai 2015,

consulté le 21 février 2020. URL : http://journals.openedition.org/ere/533 ; DOI : 10.4000/ere.533 


\title{
Quand les adolescents passent à l'action en matière de santé environnementale
}

\author{
Nathalie Robitaille
}

1 Les adolescents constituent une force vive de notre société. Ils n'ont toutefois pas toujours conscience ou confiance en leurs capacités d'acteurs. Les adolescents d'aujourd'hui sont pourtant les décideurs de demain. Ils devront faire face à des enjeux de santé environnementale de plus en plus complexes et prendre des décisions qui allient des dimensions politiques, éthiques et sociales relativement à ces enjeux.

2 L'adolescence correspond à une période riche en transformations et en quête d'autonomie, d'appartenance, de reconnaissance ; elle se caractérise par la volonté de prendre ses propres décisions et de vivre des expériences. C'est également une période de questionnements, de préoccupations et de transitions.

3 À la lumière de certains éléments de la littérature spécialisée, cet article portera un regard critique sur la place de l'éducation relative à l'environnement (ERE) et à la santé (ES) dans le programme de l'école québécoise. Les grandes lignes d'un projet de recherche que j'ai mené seront présentées, de même que certains résultats.

\section{Préoccupations, connaissances et engagement : que savons-nous des adolescents?}

Nous savons peu de choses du rapport que les adolescents entretiennent avec leur environnement; de même, nous connaissons peu leurs préoccupations à l'égard des enjeux environnementaux, sociaux et de santé. Selon Pruneau et Chouinard (1997), l'attachement au lieu, le sens du lieu, l'identité écologique et l'identité communautaire sont des aspects déterminants d'une relation privilégiée avec l'environnement et constitueraient un prérequis à l'action environnementale. 
5 L'éducation environnementale traditionnelle mise sur une approche de transmission de connaissances. Or, les connaissances environnementales ne sont pas seules responsables du désir de passer à l'action; on observe très souvent en effet une divergence significative entre le savoir, le vouloir et l'agir. La prise de conscience ne semble pas nécessairement entraîner l'action environnementale lucide et engagée vers les changements que la situation exige (Marleau, M.-È. 2009).

Selon Jensen (2002), les connaissances environnementales ne conduisent pas en ellesmêmes à l'action environnementale ou au développement de conduites favorables à l'environnement: elles font partie d'un ensemble de conditions préalables au développement de compétences menant au changement d'attitude et à l'action.

Diane Pruneau et son équipe (Pruneau et coll., 2006) identifient des facteurs qui moduleraient et influenceraient le comportement environnemental, à savoir des facteurs cognitifs, affectifs et situationnels. Selon ces chercheurs, «le désir d'action environnementale peut être stimulé suite à un contact direct, donc émotif, avec le problème environnemental et encouragé par la réussite d'actions environnementales préalables ».

8 Jensen (2002) considère que la connaissance des moyens d'action et la capacité de construire des visions et des rêves viennent amplifier les chances de stimuler un changement de comportement, de renforcer l'intention d'agir et de développer le savoir et le pouvoir-faire. Avoir la possibilité d'agir ici et maintenant catalyserait les changements de conduite en faveur de l'environnement dans la communauté locale.

9 Selon Pruneau et coll. (2006), le fait de réussir une petite action développe le sentiment d'accomplissement et de bien-être, et stimule l'individu à entreprendre d'autres actions. Dans le même ordre d'idées, Kollmuss et Agyeman (2002) estiment que le comportement pro-environnemental serait basé sur la connaissance des problèmes, des stratégies d'action, ainsi que la perception que notre geste peut changer et/ou améliorer les choses.

10 À la lumière de ces recherches, il apparaît impératif de développer des approches pédagogiques qui soient axées sur le regard critique et la praxis, afin de permettre aux adolescents de s'approprier et d'internaliser les savoirs. En particulier, nous devons être vigilants à l'égard de l'impact psychologique que peut avoir la sensibilisation à certaines problématiques environnementales chez les adolescents. Les jeunes qui ont participé à la recherche dont traite cet article ont affirmé se sentir parfois impuissants et découragés devant la complexité de certains enjeux. Le savoir relatif à une problématique environnementale complexe peut entrâner de vives inquiétudes ainsi qu'une forme de paralysie advenant le cas où ce savoir ne serait pas supporté par une compréhension des causes et des stratégies d'action (Jensen, B.B. 2002).

\section{La place de l'ERE dans le programme de formation de l'école québécoise}

11 Le programme de formation de l'école québécoise (PFEQ), tel qu'il est conçu, laisse peu de place à l'éducation relative à l'environnement (ERE), à l'éducation à la santé (ES) et encore moins à l'éducation relative à la santé environnementale (ERSE).

Depuis 2005, année de l'implantation de la nouvelle réforme de l'école québécoise au secondaire, nous avons constaté une fragilisation de l'éducation à la santé. La 
disparition du cours de formation professionnelle et sociale (FPS), lequel offrait une plateforme pour aborder des thèmes tels que la sexualité, les enjeux sociaux et les relations interpersonnelles, a nettement diminué l'importance accordée aux questions relatives à la santé. Le cours «Éducation physique et à la santé » n'occupe que deux heures trente minutes par période de neuf jours, et la santé n'y est abordée que dans le contexte d'activités physiques et de saines habitudes de vie. Or l'éducation à la santé n'est plus seulement la responsabilité des professionnels de la santé, mais elle est devenue une responsabilité partagée entre tous les enseignants. Mais ceux-ci se sentent-ils suffisamment formés et compétents pour s'acquitter de cette tâche ? À la lumière des échanges tenus avec plusieurs d'entre eux, il semblerait que non.

Par ailleurs, dans le programme de science et technologie de l'environnement, l'environnement n'est abordé que sous l'angle de thématiques telles que les changements climatiques, l'eau, la déforestation, l'énergie, les matières résiduelles et la production alimentaire. Or ces thématiques sont malheureusement traitées en silo. Rarement les jeunes sont mis en situation d'apprentissage axée sur les réalités qu'ils les vivent et les invitant à la praxis.

14 Afin de combler cette lacune, il importe de s'intéresser aux représentations et aux préoccupations relatives à la santé et l'environnement des adolescents, ainsi qu'à la perception qu'ils ont de leur pouvoir-agir? Certains chercheurs (Meinhold et Malkus, 2005) croient que lorsque les adolescents s'engagent et se sentent bien à l'égard de leurs gestes et conduites, leur niveau d'estime de soi et leur sentiment d'auto-efficacité augmentent.

15 L'éducation relative à la santé environnementale (ERSE) apparaît comme une fenêtre porteuse pour transformer le rapport à l'environnement et à la santé des jeunes en rapprochant ces réalités de leur vie quotidienne. L'approche active et participative privilégiée en ERSE, favorise une co-construction de savoirs ainsi que l'élaboration de solutions proactives individuelles et collectives aux problématiques de santé environnementale (Sauvé et Godmaire 2009). L'apprentissage au cœur de l'action nourrit le sentiment de pouvoir-agir (empowerment) chez l'adolescent qui est en quête de sens, de sentiment d'appartenance et de développement de l'estime de soi.

\section{Une démarche éducative en santé environnementale auprès des adolescents}

16 Adoptant un paradigme interprétatif et critique, la recherche que nous avons menée a tenté de replacer les élèves au cœur de leur environnement. Il s'agit de développer des savoirs pédagogiques permettant de les inciter à développer un regard critique afin d'appréhender la complexité de ces réalités, et de les inviter à transformer les réalités socio-environnementales et de santé qui les concernent. Nous souhaitons contribuer au développement d'une pédagogie qui favorise l'émergence d'un vouloir-agir et d'un pouvoir-agir en faveur d'une relation plus harmonieuse entre la santé et l'environnement.

17 C'est donc dans une perspective participative, réflexive et praxique que nous avons mené une recherche-développement auprès de 58 élèves de $4 \mathrm{e}$ secondaire d'un établissement affilié au réseau des Écoles d'éducation internationale, à McMasterville en Montérégie (Québec) ${ }^{1}$. La recherche a été rendue possible grâce à la précieuse 
collaboration de l'enseignant ${ }^{2}$ en sciences et technologie qui croyait en l'importance d'aborder les notions de santé et d'environnement dans une approche systémique et holistique. Il s'agissait de concevoir, développer, d'expérimenter et d'évaluer un microprogramme d'éducation à la santé environnementale auprès des jeunes.

Les questions qui ont guidé cette recherche s'articulent ainsi : Comment les adolescents perçoivent-ils le lien entre l'environnement et la santé?; Quelles sont leurs préoccupations à l'égard de la santé et de l'environnement?; Quels sont les facteurs qui suscitent ou qui limitent leur désir d'agir ?; Comment perçoivent-ils leur pouvoiragir en faveur des enjeux de santé environnementale? Quelles approches et stratégies pédagogiques sont appropriées afin de stimuler le désir d'engagement et le pouvoiragir des adolescents en faveur des enjeux de santé environnementale?

Cette recherche, inspirée des travaux de Diane Pruneau (2003), s'intéresse aux représentations et aux préoccupations des adolescents en ce qui concerne la santé environnementale, à leur savoir-être, leur savoir-agir ainsi qu'à leur sentiment de pouvoir-agir. Il s'agit d'enrichir de contribuer au développement d'interventions éducatives appropriées dans le champ de l'éducation relative à la santé environnementale, en offrant une proposition pédagogique validée, soit un microprogramme en éducation relative à la santé environnementale.

Le lien santé-environnement est malheureusement trop souvent abordé dans une perspective négative : l'environnement est perçu comme un agresseur. Afin d'aborder la santé environnementale de façon proactive et positive, nous avons utilisé l'approche écosystémique de la santé où les apprenants participent activement à l'élaboration de savoirs, au développement de l'esprit critique; ils sont incités à faire preuve de curiosité, d'autonomie, de vouloir, de savoir et de pouvoir-faire (Sauvé et Godmaire, 2004) et à devenir des acteurs et des moteurs de changement (Lebel, 2003) dans leur milieu.

21 C'est à travers une diversité de rencontres et d'activités que les élèves ont été invités à s'engager dans un projet collectif dans, par et pour la santé environnementale. Le microprogramme en éducation relative à la santé environnementale a été créé puis mis à l'essai auprès d'adolescents dans le but de développer un vouloir et un pouvoir-agir en faveur de la santé environnementale. La recherche-développement que nous avons menée poursuit les objectifs généraux suivants :

- Caractériser le rapport des adolescents à l'environnement et à la santé ;

- Identifier les préoccupations qu'ont les adolescents à l'égard de la santé et de l'environnement ;

- Cerner le désir d'agir ainsi que la perception qu'ont les adolescents de leur capacité d'agir ;

- Cerner les facteurs favorisant ou limitant ce désir et ce pouvoir-agir ;

- Valider la pertinence d'un microprogramme en éducation relative à la santé environnementale dans le développement d'un vouloir et d'un pouvoir-agir chez les adolescents ;

- Proposer une démarche éducative validée qui soit catalyseur d'intérêt, de changement et d'engagement en faveur de l'environnement et de la santé.

Parmi les principales activités proposées dans le microprogramme, nous retrouvons les suivantes :

- Introduction à l'environnement accompagnée d'une activité de reboisement ;

- «Si le monde était un village de 29 personnes... » - jeu de rôle ;

- L'eau, source de vie, de mort et de conflits ; 
- La pollution sous toutes ses formes ;

- La santé et l'environnement : une relation indissociable !;

- La relation intergénérationnelle ;

- Les cosmétiques et la santé ;

- Le passage à l'action!

\section{Objectifs et méthodologie de la recherche}

Cette recherche-développement poursuivait les objectifs suivants :

- Caractériser le rapport des adolescents à l'environnement et à la santé ;

- Identifier les préoccupations qu'ont les adolescents à l'égard de la santé et de l'environnement ;

- Cerner le désir d'agir ainsi que la perception qu'ont les adolescents de leur capacité d'agir ;

- Cerner les facteurs favorisant ou limitant ce désir et ce pouvoir-agir ;

- Valider la pertinence d'un microprogramme en éducation relative à la santé environnementale dans le développement d'un vouloir et d'un pouvoir-agir chez les adolescents ;

- Proposer une démarche éducative validée qui soit catalyseur d'intérêt, de changement et d'engagement en faveur de l'environnement et de la santé.

La démarche comportait quatre phases : 1) le diagnostic de la situation problème que nous souhaitons contribuer à résoudre; 2) la conception d'un microprogramme d'éducation relative à la santé environnementale; 3) la mise à l'essai du microprogramme ; 4) l'évaluation et la rétroaction en vue de l'amélioration et de la diffusion du microprogramme. Cet article se penche sur certains résultats relatifs à l'étape 3) de la recherche-développement.

La diversité des stratégies de collecte de données - telles que l'observation participante, le questionnaire, l'entrevue semi-dirigée et le world café - a permis d'enrichir le bagage d'informations et d'assurer une triangulation des données. La mise à l'essai du microprogramme s'est échelonnée pendant une année scolaire, soit du mois de septembre au mois de juin. Un questionnaire a été soumis aux élèves au début de la mise à l'essai du microprogramme et à la fin. Les élèves ont également participé à des entrevues semi-dirigées au début et à la fin de la mise à l'essai. Le world café, qui consiste à favoriser le dialogue autour de questions précises dans une dynamique active où les gens se déplacent afin de multiplier les interactions, a permis de valider les résultats grâce à l'utilisation de questions similaires à celles du questionnaire et du guide d'entrevues.

\section{Quelques résultats}

Rappelons que le but de cette recherche était de contribuer à l'enrichissement du champ théorique et de pratique de l'éducation relative à la santé environnementale appliquée au contexte scolaire et aux adolescents. Le défi est de favoriser la transformation des représentations initiales et le développement de l'intention d'agir individuellement ou collectivement en faveur de la santé environnementale.

Les résultats mettent en lumière une certaine évolution ou transformation des représentations de l'environnement et de la santé chez les élèves qui ont participé à la 
recherche. Selon la typologie des représentations de l'environnement développée par Lucie Sauvé (2003), on remarque que l'environnement nature (forêt, nature, plantes, rivières, etc.) est initialement très présent, de même que l'environnement projet communautaire (recyclage, compostage, groupes, sensibilisation, etc.). Aucun élève n'a fait de lien avec la santé. En fin de recherche, la vision systémique se développe et le "tout ce qui m'entoure» est davantage explicité. L'environnement nature occupe toujours le premier rang des représentations et l'environnement "milieu de vie » s'impose à son tour. L'environnement problème est exprimé, mais n'occupe pas une place centrale.

En ce qui a trait aux représentations de la santé, au début de la recherche, l'image était plutôt négative: maladies, asthme, embonpoint, souffrance, problèmes, cancer, blessures, ambulance, etc. Pour ces jeunes, l'éducation à la santé s'est souvent résumée aux thématiques de l'alimentation saine et à l'importance de faire de l'activité physique et de dormir suffisamment. Il ne faut donc pas se surprendre que ces éléments de représentations soient exprimés ici. Le lien avec l'environnement a trait à l'impact des facteurs environnementaux sur la santé humaine, encore une fois dans une perspective d'agression.

À la fin de la recherche, les jeunes avaient une image plus proactive et plus globale de la santé. La notion de maladie était moins importante et les jeunes parlaient d'environnement et non de facteurs environnementaux. La préoccupation envers les générations futures était très présente dans leur discours : ils se sentaient responsables d'agir maintenant.

\section{Comment les jeunes perçoivent-ils le lien entre la santé et l'environnement?}

Tous les des élèves comprennent et expliquent certains aspects du lien entre la santé humaine et l'environnement.

La santé dépend de deux aspects importants : le milieu de vie et les habitudes de vie. Par exemple, les problèmes de santé des populations vivant près des centrales nucléaires, les problèmes pulmonaires des travailleurs en contact avec l'amiante, les cas d'asthme plus fréquents à cause de la qualité de l'air.

Il y a différentes "santé »: santé mentale et physique. L'environnement est une bonne place pour se changer les idées, faire du sport, avoir du plaisir, ce qui est très bien pour la santé mentale. Nous pouvons courir pour avoir une santé physique excellente.

Ton environnement a un grand impact sur ta santé même si tu ne peux pas toujours voir le lien clairement.

Le fait de voir la nature, la forêt, des arbres, apaise les gens.

La santé de l'homme dépend étroitement de celle de l'environnement car il vit et interagit continuellement avec lui.

Les décisions que nous prenons influencent l'environnement, qui lui, va influencer notre santé.

31 Bien que les connaissances ne soient pas les seules responsables du désir d'agir, nous nous sommes tout de même intéressées à l'acquisition de connaissances dans une approche participative lors de la mise à l'essai du microprogramme (Figure 1). À la lumière des réponses des élèves aux questionnaires et aux entrevues semi-dirigées, on 
observe que l'acquisition de connaissances relatives à la santé environnementale a été notable.

Figure 1 : Évolution des connaissances en cours de microprogramme

\section{AS-TU LES CONNAISSANCES NÉCESSAIRES ?}

Début de la recherche

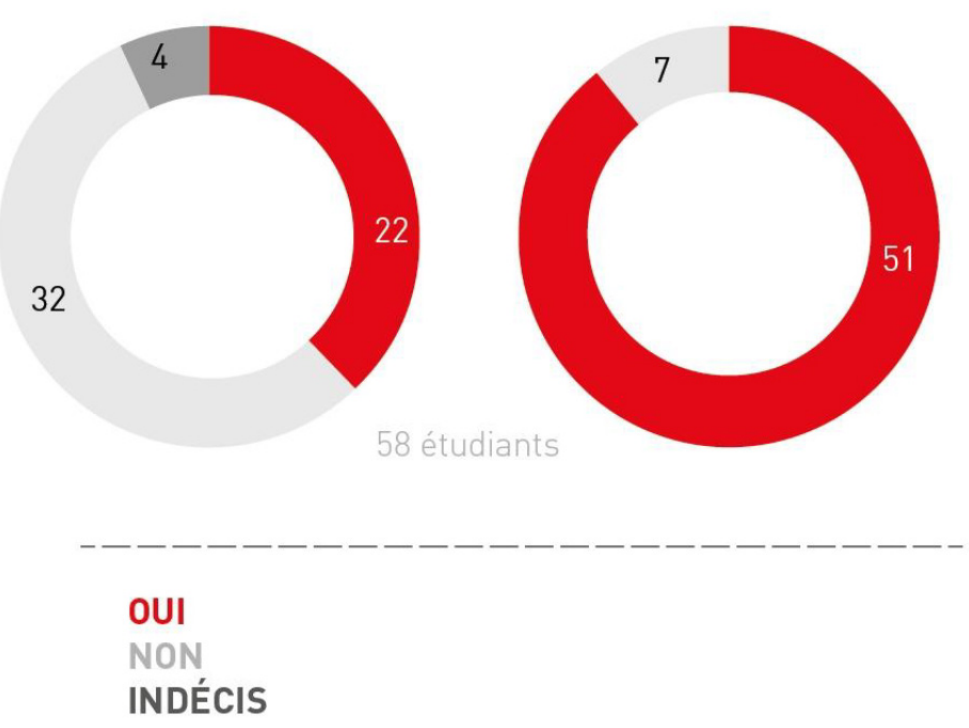

Et le désir d'agir ? Le microprogramme a-t-il permis de le renforcer?

Les élèves se disent plus conscients et engagés qu'au début (Figure 2). Avant leur participation au microprogramme, ils s'engageaient en faveur de l'environnement, mais, maintenant, ils savent pourquoi ils le font. Ils croient en ce qu'ils disent, font des débats entre eux, sont conscients des impacts et cela les motive. Ils mentionnent que le fait de poser un geste concret accroît le désir d'agir ; ils comprennent les bénéfices pour la santé et l'environnement. De plus, "s'engager collectivement en faveur d'un projet d'envergure, c'est le fun!» 
Figure 2 : Évolution du désir d'agir en cours de microprogramme

\section{ENVIE D'AGIR ?}

Début de la recherche

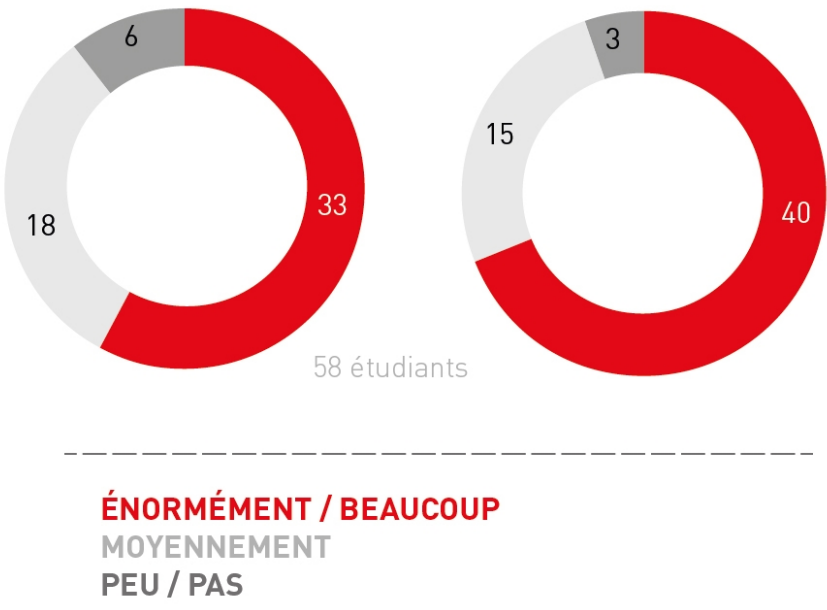

34 La participation au microprogramme et la connaissance des moyens d'action font en sorte que les élèves se disent plus engagés (Figure 3) : "Je lis les ingrédients"; "J'ai changé de produits de cosmétiques"; "Je sensibilise les gens autour de moi ": "J'essaie d'implanter le compostage à la maison »; "Je n'achète plus d'eau embouteillée ». 
Figure 3 : Évolution du pouvoir-agir en cours de microprogramme

\section{AS-TU LE POUVOIR D'AGIR ?}

Début de la recherche

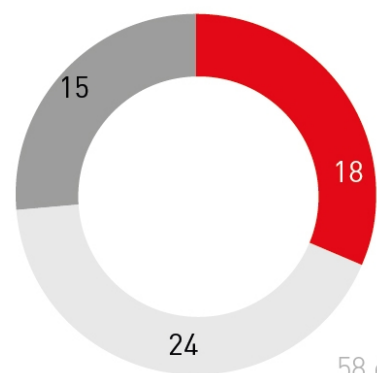

58 étudiants
Fin de la recherche

16

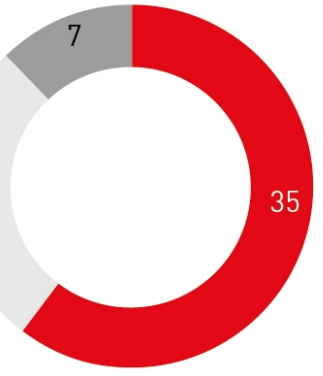

ÉNORMÉMENT / BEAUCOUP

MOYENNEMENT

PEU / PAS

Les résultats relatifs au sentiment de compétences sont incontestablement les plus révélateurs, surtout dans la période de construction d'estime de soi qu'est l'adolescence (Figure 4). Un élève se reconnaît comme un agent de changement et, un modèle ; il a conscience d'avoir le pouvoir d'influencer autant ses parents, sa famille que ses amis. D'autres disent qu'ils possèdent les arguments pour sensibiliser les pairs et les inciter à passer à l'action. 


\section{TE SENS-TU COMPÉTENT ?}

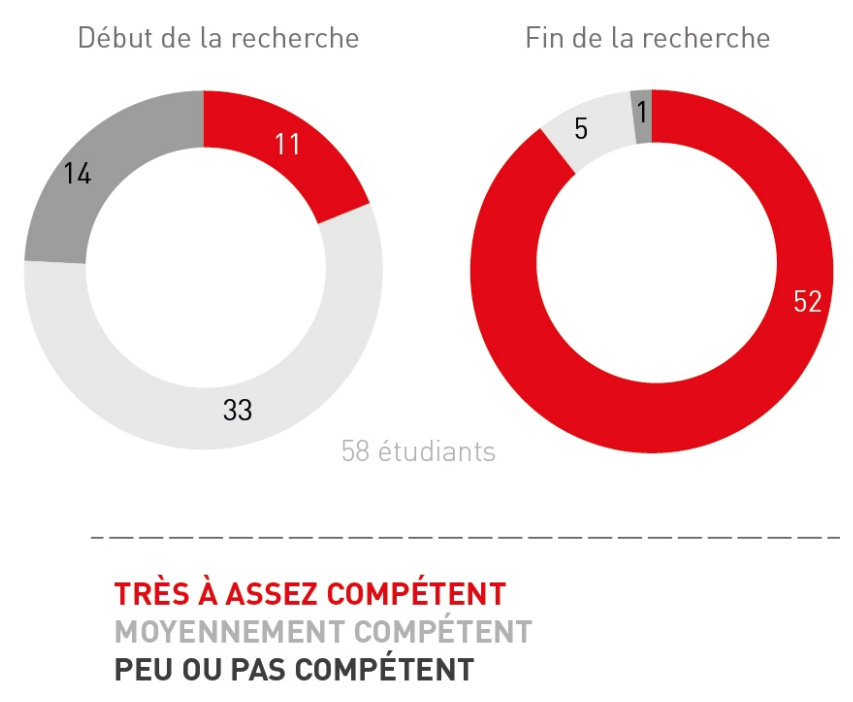

\section{Éléments de discussion}

Plusieurs chercheurs (Musser et Diamond, 1999 ; Kasapoglu et Turan, 2008) sont d'avis que l'école et les parents ont un rôle prédominant dans le développement d'attitudes et de conduites en faveur de l'environnement chez les enfants. Or peu d'études se sont intéressées à ce rôle.

Selon Hart (2000), pour améliorer notre compréhension du processus de participation des enfants à des activités et à des projets en éducation relative à l'environnement, il importe d'abord de s'intéresser à leurs perceptions et leurs réflexions à l'égard de l'environnement? Il faut également aller au-delà de la simple observation et explorer plus en profondeur les situations d'enseignement et d'apprentissage; il faut aussi engager des discussions avec les enseignants et les élèves afin de connaître le sens qu'ils donnent aux expériences vécues.

L'école peut être un milieu propice à la conscientisation de la relation entre la santé et l'environnement, ainsi qu'un milieu favorable pour stimuler la participation via des activités appropriées afin de susciter le développement d'un savoir et d'un pouvoir-agir (Kasapoglu et Turan, 2008).

Le microprogramme que nous avons développé en éducation relative à la santé environnementale (ERSE) semble proposer des pistes pour renforcer le pouvoir-agir des jeunes et leur participation à l'égard des problématiques de santé environnementale qui caractérisent leur milieu de vie. Selon l'enseignant en sciences de $4 \mathrm{e}$ secondaire, les élèves sont prêts à prendre conscience de leur interaction avec l'environnement et de la place qu'ils peuvent y prendre. Toujours selon lui, le microprogramme nous a permis d'aller plus loin, d'être plus concrets, de les impliquer dans l'environnement; ils deviennent des acteurs. Les 
activités concrètes leur ont permis de comprendre mieux leurs choix d'action, d'adopter de nouvelles façons d'agir et d'en être fier! L'apprentissage dans l'action, par contact direct est une dimension importante, car on trouve ici une dimension affective.

Dans son analyse faite sous l'angle du baccalauréat international, la conseillère pédagogique que nous avons interrogée, considère que le microprogramme touche à tous les domaines généraux de formation (DGF), notamment celui du « vivre ensemble et citoyenneté ». " Dans ce microprogramme, il y a un cycle de savoir-être, de savoiragir et de pouvoir-agir qui correspond tout à fait aux aires d'interaction qui nous apparaissent importantes pour la formation des jeunes ${ }^{3}$. "Selon celle-ci, il importe d'inciter les jeunes à poser un regard critique sur leur rapport à l'environnement et d'offrir des pistes de solutions, comme le fait le microprogramme que nous avons conçu et expérimenté. L'enthousiasme manifesté par les divers acteurs du milieu de l'éducation nous incite à croire que nous avons comblé le besoin de faire vivre des expériences signifiantes et engageantes aux élèves et que nous avons suscité de l'intérêt pour l'éducation relative à la santé environnementale !

Nous croyons que le fait d'offrir aux jeunes l'opportunité de s'engager collectivement et concrètement dans un projet qui les interpelle et qui les concerne devient un tremplin pour l'engagement sincère et durable. Le microprogramme que nous avons conçu et expérimenté nous semble transférable dans la perspective où il permet aux jeunes, indépendamment de leur milieu social, économique, culturel ou environnemental, de redéfinir le lien entre la santé et l'environnement, de poser un regarder critique sur les enjeux qui façonnent leur quotidien et d'acquérir des savoirs et d'adopter un agir contribuant à un rapport plus harmonieux à l'égard de l'environnement.

\section{BIBLIOGRAPHIE}

Gouvernement du Québec (2003). Programme de formation de l'école québécoise. Enseignement secondaire premier cycle. Un programme de formation pour le XXIe siècle. Québec : Ministère de l'Éducation, du Loisir et du Sport du Québec.

Hart, P. (2000). Searching for meaning in children's participation in environmental education. In Jensen, B., Schnack, K., Simovska, V. Critical environmental and health education. Research issues and challenges (p. 7-28). Copenhagen : Research center for environmental and health education. The Danish University of education.

Jensen, B.B. (2002). Knowledge, Action and Pro-environmental Behaviour. Environmental Education Research. vol. 8 no 3, p. 325-334.

Kasapoglu, A., Turan, F. (2008). Attitude-behaviour relationship in environmental education : A case study from Turkey. International Journal of Environmental Studies. vol. 65, no 2, p. 219 231.

Kollmuss, A., Agyeman, J. (2002). Mind the gap : Why do people act environmentally and what are the barriers to pro-environmental behaviour? Environmental Education Research. Vol 8, No. 3. P. $x x-x x$ 
Lebel, J. (2003). La santé : une approche écosystémique. Ottawa : Centre de recherches pour le développement international.

Marleau, M. È. (2009). Des liens à tisser entre la prise de conscience et l'action environnementales. Éducation et francophonie, 37(2), 11-32. Meinhold, J. L., Malkus, A. J. (2005) Adolescent environmental behaviors. Can Knowledge, Attitudes, and Self-Efficacy Make a Difference ? Environment and Behavior, vol 37, No 4, p. 511-532.

Musser, L., Diamond, K. (1999). The children's attitudes toward the environment scale for preschool children. Journal of environmentla education. vol. 30, no 2, p. 23.

Pruneau, D. et Chouinard, O. (1997). Un modèle d'intervention pédagogique qui favorise la relation personne/groupe/social/environnement. In M. Hrimech et F. Jutras (eds), Défis et enjeux de l'éducation dans une perspective planétaire. Sherbrooke : Éditions du CRP, 107 - 123.

Pruneau, D., Doyon, A., Langis, J., Vasseur, L., Martin, G., Ouellet, E., Boudreau, G. (2006). L'adoption de comportements environnementaux : motivations, barrières et facteurs facilitants. Article qui a été publié en anglais sous le nom de « The Process of Change Experimented by Teachers and Students when Voluntarily Trying Environmental Behaviours » dans Applied Environmental Education and Communication, 5 (1). En ligne, consulté le 6 avril 2012. www.espace-ressources.uqam.ca/images/Documents/Recherche/Articles_interet/ D.PRUNEAU(2006).pdf

Pruneau, D., Langis, J., Richard, J.-F., Albert, G. (2003). Quand l'enseignement des sciences fait évoluer les idées des élèves au sujet de la pollution et de la santé. Vertigo - La revue électronique en sciences de l'environnement [En ligne], Volume 4 Numéro 2 | octobre 2003, mis en ligne le 01 octobre 2003 ; consulté le 17 septembre 2012. URL : http://vertigo.revues.org/4539; DOI : $10.4000 /$ vertigo.4539.

Sauvé, L. (2003). L'éducation relative à l'environnement : une diversité de perspectives. Module 1. Programmes d'études supérieures - Formation en éducation relative à l'environnement Francophonie internationale. Montréal :, Les publications ERE - UQAM, Université du Québec à Montréal - Collectif ERE-Francophonie.

Sauvé, L. et Godmaire, H. (2004). Environmental health education : participatory holistic approach. Ecohealth. vol. 1 suppl. 2.

Sauvé, L. et Godmaire, H. (2009). Pour une éducation relative à la santé environnementale : une approche écosystémique et participative. Liaison Énergie-Francophonie. Institut de l'énergie et de l'environnement de la francophonie, numéro 82, 61-70

\section{NOTES}

1. L'École d'éducation internationale, située à McMasterville en Montérégie, offre une triple diplomation aux élèves, soit le diplôme du ministère de l'Éducation, du Loisir et du Sport, le DÉSI (diplôme d'éducation internationale) et le certificat du Programme de premier cycle du secondaire, émis par le Baccalauréat international (IB). Afin d'obtenir leurs diplômes de l'IB, les élèves doivent s'engager activement et bénévolement dans la communauté, durant minimalement 70 heures au cours des cinq années du secondaire. http://eei.csp.qc.ca/index.html 2. Je tiens à remercier l'enseignant Éric Gourdon pour son ouverture, son enthousiasme et sa précieuse collaboration. Merci à Marie Beauregard, conseillère pédagogique, pour son analyse critique du microprogramme. La recherche a également été rendue possible grâce à la confiance que m'ont accordée l'équipe-école ainsi que le conseil d'établissement. 
3. École d'éducation internationale - Les aires d'interaction : http://eei.csp.qc.ca/html/ a_propos.html\#interaction

\section{INDEX}

Keywords : environmental education, environmental health, curriculum, teenager, commitment Mots-clés : éducation relative à l'environnement, santé environnementale, curriculum, adolescent, engagement

Index géographique : Québec

\section{AUTEUR}

\section{NATHALIE ROBITAILLE}

Candidate à la maîtrise en science de l'environnement à l'Université du Québec à Montréal. Elle est également conseillère senior pour l'organisme à but non lucratif Synergie Santé Environnement où elle accompagne les établissements de santé à réduire leur empreinte environnementale et à améliorer leurs pratiques en santé environnementale. Pendant 23 années, Nathalie a travaillé dans le domaine de la santé à titre d'inhalothérapeute au Centre hospitalier universitaire de Montréal. 